\title{
Avantages et Faisabilité de l'Épandage Forestier des Boues Résiduaires
}

\author{
par
}

\author{
Yvon Grenier ${ }^{1}$ et Denis Couillard ${ }^{2}$
}

\section{Résumé}

Une revue de littérature permet de vérifier que l'épandage des boues résiduaires en forêt est une opération techniquement réalisable, puisque des machines spécialisées ont été mises au point. Les épandages pourraient faire partie d'un plan d'aménagement forestier où cette forme particulière de fertilisation serait couplée à des opérations sylvicoles. Des augmentations de croissance en hauteur de l'ordre de $350 \%$ après 4 mois chez des semis et de $250 \%$ après 3 ans chez de jeunes plants sont des arguments en faveur de la fertilisation forestière à l'aide de boues résiduaires. La seule utilisation des terres agricoles ne serait peut être pas suffisante pour écouler la production totale de boues d'une localité; les aires boisées viennent donc en renfort afin de réaliser le maximum de recyclage de cette nouvelle ressource que sont les boues résiduaires.

Mots-clés : aménagement forestier, boue résiduaire, fertilisation forestiere, machinerie d'épandage.

\begin{abstract}
A literature review brings some evidences that wastewater sludge disposal in the forest is technically feasible since specialized machines have been designed for this purpose. Sludge utilization could be a part of a forest management plan where this particular form of fertilization would take place during silviculture operations. Additional height yields of $350 \%$ after 4 months growth with seedlings and $250 \%$ after 3 years with young plants have been observed showing that forest fertilization with wastewater sludge is beneficial. The use of agricultural land only for disposal of the sludge production of a municipality may be inadequate; wooded areas can be used as complementary surfaces to maximize the recycling of this new resource, the sludge.
\end{abstract}

Key words: forest fertilization, forest management, spreading machinery, wastewater sludge.

\section{Problématique des boues d'épuration au Québec}

Le gouvernement du Québec, par l'entremise du ministère de l'Environnement, a mis sur pied un programme d'assainissement des eaux usées. Commencé en 1978 et échelonné sur une dizaine d'années, ce programme touche toutes les régions du Québec (Couillard et al., 1986; Gouvernement du Québec, 1984). Avec des investissements de l'ordre de 6,7 milliards de dollars, soit 4,7 milliards pour le volet municipal, 1,5 milliards pour le volet industriel et 0,5 milliard pour le volet agricole (Gouvernement du Québec, 1985b; Crowley et al., 1984), le gouvernement veut améliorer la sécurité d'approvisionnement en eau, protéger ou redonner les usages de loisirs et de récréation et assurer des conditions propices à un milieu de vie aquatique équilibré (Gouvernement du Québec, 1985a).

\footnotetext{
Ministère de l'Énergie et des Ressources, Service de la Recherche Appliquée 2700, rue Einstein. Sainte-Foy, Québec, Canada G1P 3W8

Institut national de la recherche scientifique (INRS-Eau) - C.P. 7500, 2700 rue Einstein - Sainte-Foy, Québec, Canada G1V 4C7
}

Les usines qui traitent les eaux usées permettent le rejet, dans le milieu, d'une eau de qualité acceptable, mais produisent un résidu appelé boue résiduaire, ou boue. À ce chapitre, le Québec est en retard: en 1984, seulement 10\% de ses eaux municipales étaient traitées, alors que l'Ontario et l'ensemble du Canada en traitaient respectivement $84 \%$ et $58 \%$ à cette même époque (Gouvernement du Québec, 1984). En 1987, 29\% des eaux municipales québécoises (incluant celles de la CUM) étaient traitées (Gouvernement du Québec, 1987). L'élimination et le traitement des boues résiduaires accaparent jusqu'à $50 \%$ des coûts de fonctionnement d'une usine d'épuration (Lester et al., 1983) et représentent l'un des plus grands problèmes auquel les ingénieurs sanitaires doivent faire face aujourd'hui (Hecht et al., 1975). À ce sujet, le rapport Dagenais rapporte qu'une politique de gestion des boues résiduaires doit être établie le plus tôt possible pour le Québec (Gouvernement du Québec, 1986a).

La quantité de boue produite dépend des traitements qu'on fait subir aux eaux usées et de la population qui est desservie par l'usine d'épuration (Couillard, 1988). En général, les auteurs s'accordent à dire que la production quotidienne oscille entre 
500 (Vergès, 1984; Anred, 1983) et 1000 kg de boue sèche par 10000 habitants (Environnement Canada, 1985; Webber, 1984; Hornbeck et al., 1979; Sabey et Hart, 1975).

Au Québec même, 900 municipalités sont visées par le programme d'épuration des eaux (Gouvernement du Québec, 1985b), ce qui représente 5,2 millions de personnes (Giroux, 1986; Gagnon, 1985). On peut donc s'attendre à produire entre 100000 et 200000 tonnes de boues sèches annuellement, ce qui représente une quantité assez considérable de boue à gérer.

II faut être conscient que, jusqu'à maintenant, les coûts d'élimination des déchets n'étaient pas représentatifs de la réalité. Les ordures étaient simplement mises de côté et ce, à peu près n'importe où. Cependant, les choses changent (Couillard, 1983) et il est de plus en plus admis que des frais sont imputables à la disposition des rebuts; il y a plus de contrôles et les règlements sont plus stricts. Ainsi, puisque ce sera de plus en plus coûteux pour se débarrasser des déchets, les avantages du recyclage deviennent plus tangibles en raison de la valeur économique qui en découle.

La ministère de l'Environnement du Québec est en faveur du recyclage et de la récupération des déchets, incluant les boues résiduaires. Le mode de valorisation privilégié est l'épandage sur les terres agricoles (Gouvernement du Québec, 1984; 1983). Ce moyen est aussi suggéré par Environnement Canada (1985) et l'Agence de protection de l'environnement américaine (EPA, 1979) pour disposer des boues résiduaires. Le recyclage est économique et plus écologique, en général, que la simple disposition (Baldwin et al., 1983; Sabey et Hart, 1975) car il permet de récupérer une partie des frais reliés à l'élimination des boues.

Cependant, trois problèmes majeurs peuvent déjà être identifiés quant au mode de gestion retenu. Premièrement, il existe au Québec des régions qui seront dotées d'usines d'épuration des eaux et où il n'y a pas ou peu d'agriculture. Deuxièmement, ce ne sont pas toutes les cultures qui peuvent recevoir des boues. À ce sujet, le MAPAQ et le MENVIQ interdisent l'épandage de boues sur des cultures maraîchères; les cibles potentielles se ramènent donc au maïs (maïs grain ou d'ensilage, ce qui exclut le maïs pour consommation humaine), aux céréales et aux prairies (Giroux, 1987). Pour l'épandage en prairie, il y a des règles qui régissent les périodes d'attente avant que le pâturage des animaux domestiques puisse être de nouveau pratiqué (Webber, 1984). Par ailleurs, les régions agricoles produisent des lisiers et fumiers qui sont épandus sur les terres et les boues pourraient entrer en compétition avec les déchets animaux pour les surfaces d'épandage. De plus, les agriculteurs ne sont pas obligés de recevoir des boues et le feront sur une base volontaire; il est donc possible que près des grands centres, surtout, il y ait un surplus de boues "destinées à l'agriculture". Troisièmement, malgré de nombreuses études citant le peu de risques environnementaux associés à la présence d'organismes pathogènes, de composés synthétiques toxiques et de métaux lourds dans les boues (Webber, 1984; EPA, 1983), les craintes reliées à leur utilisation en agriculture sont omniprésentes, du fait que les cultures produites sont ultimement destinées à la consommation humaine.

Ainsi, d'autres voies de valorisation doivent être recherchées et la filière forestière pourrait amener une solution (Couillard et Grenier, 1987; Grenier et Couillard, 1987).

\section{Exemples de fertilisation forestière par les boues résiduaires}

La foresterie a deux avantages indéniables sur l'agriculture en ce qui concerne la valorisation des boues. Premièrement, les surfaces forestières sont distribuées à la grandeur du territoire québécois. Deuxièmement, puisque les produits forestiers (ligneux) ne font pas partie de la chaine alimentaire humaine, les risques de transmission de substances indésirables (métaux lourds, composés synthétiques, etc.) sont écartés (Mclntosh et al., 1984; Webber, 1984; EPA, 1983; Fiskell et al., 1984; 1982; Riekerk, 1982; Le Tacon et al., 1979; Pommel, 1979; Sidle et Kardos, 1977).

A l'opposé des cultures agronomiques, la fertilisation des forêts naturelles ou artificielles n'est pas essentielle en soi pour leur croissance. Généralement, les surfaces boisées ne sont pas fertilisées (Crites, 1984). Cependant, la production de matière ligneuse est augmentée quand cette activité est pratiquée (Cole, 1981; Morrison, 1974). Les fertilisants accélèrent la croissance surtout en diamètre (Brockway et al., 1979; Möller, 1974), chez la plupart des espèces forestières (Vézina et Roberge, 1981; Gagnon, 1974). Ainsi, la fertilisation pourrait être un outil adéquat lorsqu'on désire tirer plus de ressources du milieu sans devoir agrandir le territoire d'exploitation, ou encore pour la production de biomasse en courtes rotations (Jones et Grant, 1983), ce qui exige des fertilisants (Miller, 1983; Blake et Raitanen, 1981). De plus, l'intensité croissante des récoltes, en arbres entiers par exemple, signifie nécessairement un plus grand enlèvement des éléments nutritifs du site (Hornbeck et Kropelin, 1982; Patrick et Smith, 1975).

Les forêts croissant sur les sols les plus pauvres sont les plus susceptibles de réagir positivement à la fertilisation (Roberge et al., 1980). Le facteur important à considérer est l'écart qui existe entre le capital en éléments nutritifs présents et assimilables dans le sol et ce qui est ajouté artificiellement en fertilisants chimiques ou organiques.

Les fertilisants chimiques coûtent cher; il serait donc intéressant de leur trouver des substituts.

Au Québec, Gagnon (1972; 1974) a appliqué 560 kg/ha de boues digérées anaérobies (base sèche) dans une plantation d'épinettes blanches (Picea glauca [Moench] Voss.) de 10 ans établie sur un sol sablonneux de faible fertilité. Le contenu en azote de cette boue était d'environ $1,5 \%$. La croissance en hauteur des arbres fertilisés a dépassé celle des témoins de $7 \%$ après 1 an, de $13 \%$ après 2 ans, de $20 \%$ après 3 ans, de $30 \%$ après 4 ans et de $38 \%$ après 6 ans.

Au Québec, Grenier (1985) et Couillard et Grenier (1987) ont appliqué jusqu'à $10285 \mathrm{~kg} / \mathrm{ha}$ de boue anaérobie à des semis de mélèze laricin (Larix laricina [Du Roi] K. Koch) croissant en serres. Après 4 mois, les semis de ce traitement affichait une croissance en hauteur supérieure de 350\% sur celle du témoin, tandis que la croissance supplémentaire en diamètre était de $210 \%$.

En Caroline du Sud, Berry et Marx (1980) ont épandu des boues digérées anaérobies dans une ancienne carrière où les horizons de surface avaient été enlevés. La quantité de boue appliquée était de l'ordre de $34000 \mathrm{~kg} / \mathrm{ha}$ (base sèche). Son contenu en azote était d'environ $2 \%$, de $1 \%$ pour le phosphore et de $0,5 \%$ pour le potassium. Des pins à l'encens (Pinus taeda 


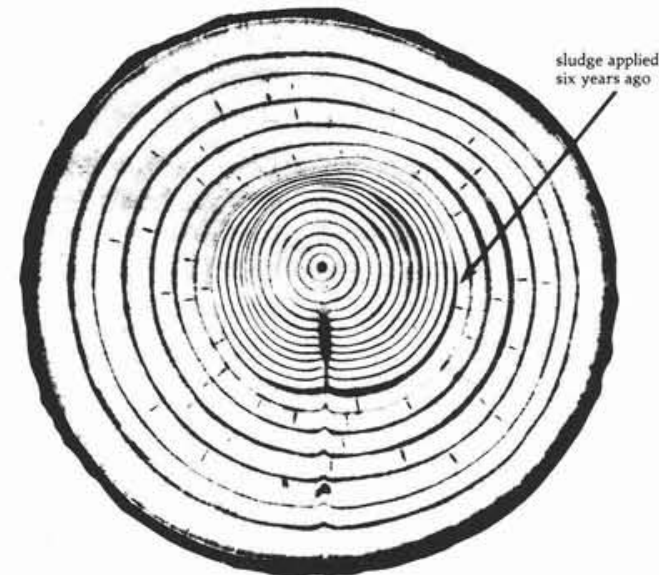

Figure 1. Reproduction d'une section transversale de Sapin Douglas ayant été fertilisé avec des boues.

L.) et des pins d'Elliott ( $P$. elliotti Engelm.) avaient été plantés sur ce site. Après 3 ans, les arbres fertilisés avaient une hauteur supérieure de $250 \%$ à celle des arbres-témoins et de $240 \%$ pour le diamètre au collet. Pour le volume des semis, les arbres fertilisés étaient 24 fois plus gros que les arbrestémoins.

En Floride, Fiskell et al. (1982) ont fertilisé des pins à l'encens ( $P$. taeda) croissant sur des sols sableux acides avec des boues digérées anaérobies provenant de Gainesville (Floride) et de Chicago (Illinois). Avec le boue de Chicago, qui contenait $5 \%$ d'azote et $2,2 \%$ de phosphore, la croissance en hauteur a été supérieure de $192 \%$ pour la dose de $4 \mathrm{t}$ / ha (4 $000 \mathrm{~kg} / \mathrm{ha})$, de $250 \%$ pour la dose de $8 \mathrm{t} / \mathrm{ha}(8000 \mathrm{~kg} / \mathrm{ha})$ et de $186 \%$ pour la dose de $16 \mathrm{t} / \mathrm{ha}(16000 \mathrm{~kg} / \mathrm{ha})$ par rapport aux arbres-témoins après 10 mois de croissance. Pour la boue de Gainesville, qui contenait 2,6\% d'azote et 2,1\% de phosphore, la croissance en hauteur a été supérieure de $130 \%$ pour la dose de $4 \mathrm{t} / \mathrm{ha}(4000 \mathrm{~kg} / \mathrm{ha})$, de $141 \%$ pour la dose de $8 \mathrm{t} / \mathrm{ha}(8000 \mathrm{~kg} / \mathrm{ha}$ ) et de $220 \%$ pour la dose de $16 \mathrm{t} /$ ha $(16000 \mathrm{~kg} / \mathrm{ha})$ après 10 mois de croissance.

Au Michigan, Brockway (1983) a fertilisé une plantation de pins blancs (Pinus strobus L.) avec des boues digérées anaérobies. La boue contenait $6 \%$ d'azote, $7 \%$ de phosphore et $0,15 \%$ de potassium. Pour la dose de $4,8 \mathrm{t} / \mathrm{ha}$ ( $4800 \mathrm{~kg} / \mathrm{ha}$ ), le diamètre à hauteur de poitrine était de $11 \%$ supérieur à celui du témoin après 2 mois de croissance et de $20 \%$ supérieur après 14 mois. Pour la dose de 19,3t/ha (19 $300 \mathrm{~kg} / \mathrm{ha}$ ), le diamètre était, après 2 mois, supérieur de $30 \%$ par rapport à celui du témoin et cette différence est portée à $47 \%$ après 14 mois de croissance.

Au Maryland, Mclntosh et al. (1984) ont fertilisé des peupliers hybrides (Populus deltoides spp. et $P$. angulata $\times P$. trichocarpa) avec des boues compostées contenant $1,36 \%$ d'azote, $2,6 \%$ de phosphore et $0,18 \%$ de potassium. Après 3 ans, la hauteur des arbres était supérieure de $207 \%$ par rapport aux témoins pour la dose de $300 \mathrm{t} / \mathrm{ha}(300000 \mathrm{~kg} / \mathrm{ha})$ et de $190 \%$ la dose de $150 \mathrm{t} / \mathrm{ha}(150000 \mathrm{~kg} / \mathrm{ha})$.

Le meilleur exemple tiré de la littérature vient de Seattle (fig. 1) (Washington) (Metro, 1983a; 1983b). Quatre usines de traitement des eaux usées acheminent leurs boues vertes à une cinquième où s'effectue une digestion anaérobie de toutes les boues produites dans l'agglomération. La boue digérée est ensuite déshydratée à un degré de siccité avoisinant $20 \%$. Puis,
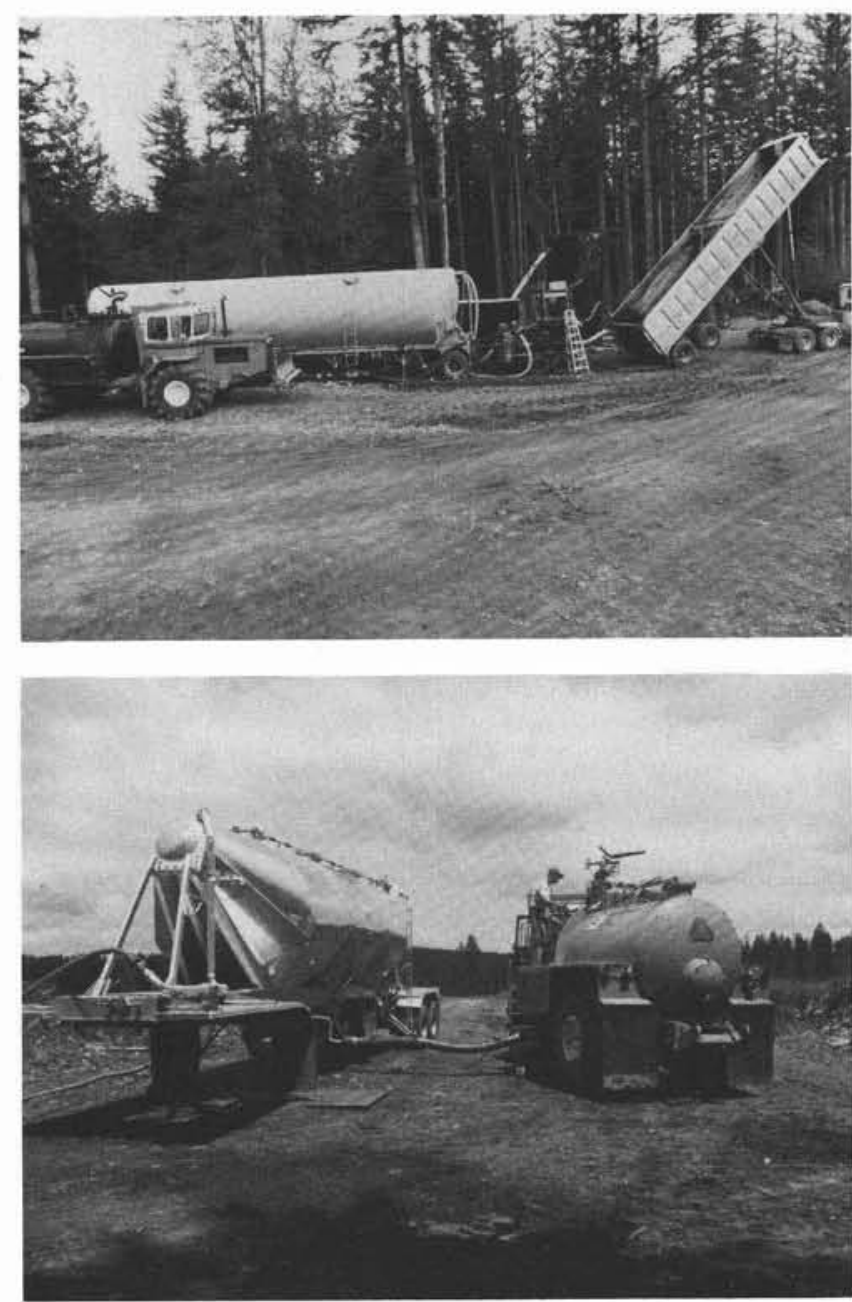

Figure 2. Transbordement de la boue.

elle quitte l'usine dans des camions-remorques pour être amenée vers les sites d'épandage où elle est préalablement entreposée. Ensuite, la boue est chargée dans des camionsciternes de type Terra-Gator. Ceux-là font la navette entre le lieu d'entreposage et les zones (figs. 2 et 3) d'épandage en appliquant la boue 50 mètres de part et d'autre du véhicule qui circule dans des chemins d'éclaircie. De telles opérations ont été réalisées avec succès à Pilchuck Tree Farm, à environ 100 $\mathrm{km}$ au nord de Seattle.

La municipalité de Seattle a fait connaître sa boue sous le nom de Silvigrow. Des résultats de croissance en diamètre allant de $200 \%$ à $400 \%$ par année sont notés (Nichols, 1983), et ce dès l'application de boue.

Le tableau 1 donne un résumé des valeurs pré-citées. Même dans l'expérience réalisée par Gagnon (1972; 1974), où la quantité de boue appliquée fut modeste, on constate quand même une amélioration non négligeable de la croissance. Cette augmentation est d'autant plus marquée que les quantités de boues (et leur contenu en azote) sont importants.

\section{Machinerie développée pour épandre les boues en forêt}

L'épandage des boues sur les terres forestières fait face à des conditions techniques différentes de celles rencontrées en valorisation agricole et ces conditions sont probablement 

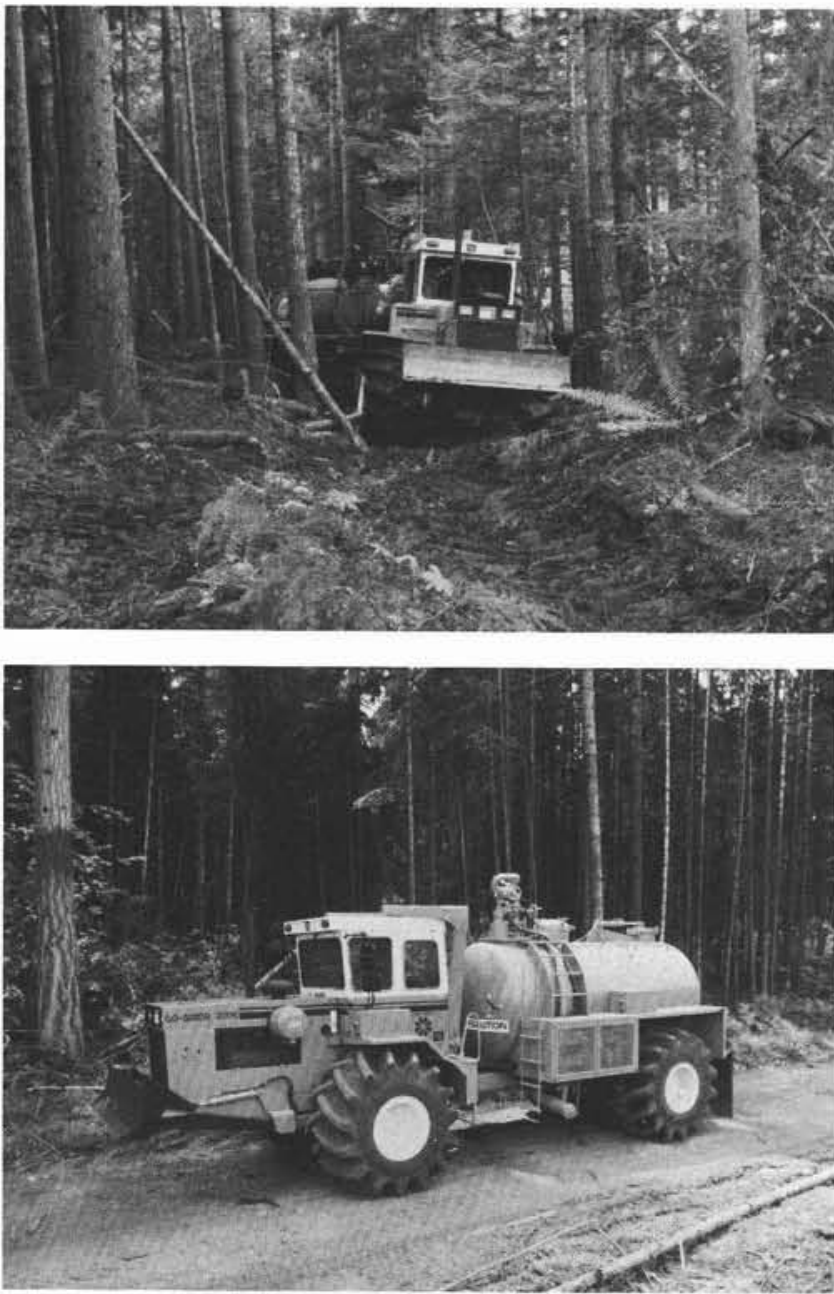

Figure 3. Véhicule Go-Gator en forêt

responsables des retards qu'a connu cette forme de recyclage. Cependant, les difficultés principales, qui sont l'accès et la circulation en forêt, ont été surmontées par la mise au point de machinerie spécialisée. A cet effet, la municipalité de Seattle et
l'Université de Washington ont fait office de pionnières en développant conjointement un véhicule apte à ce travail. Tel que rapporté par Nichols (1980), il s'agissait d'un camion de l'armée américaine à six roues motrices sur lequel étaient montés un réservoir de $7.5 \mathrm{~m}^{3}$ (2000 USG) et une pompe. Ce véhicule pouvait circuler entre les arbres et épandre la boue à l'aide d'un canon mobile.

L'idée a été reprise par des manufacturiers et aujourd'hui, il est possible de se procurer des appareils plus sophistiqués. II existe au moins deux firmes américaines qui offrent ces machines, soit Ag-Chem de Minneapolis (Minnesota) et I.M.E. de Galva (Illinois).

Un système de tuyaux et gicleurs (fig. 4) est une autre façon de faire l'épandage des boues. Ce système est adaptable et mobile. Une fois qu'un secteur a été fertilisé, le système est démonté et déménagé ailleurs. II ne nécessite pas d'investissements importants. Les gicleurs peuvent aussi être montés sur un chariot mobile, tel que rapporté par Guarino et al. (1975)

Ainsi, l'obstacle principal de la valorisation forestière, soit la circulation et l'accès en forêt a été levé. La valorisation forestière des boues d'épuration est donc techniquement possible.

\section{Épandage des boues et opérations sylvicoles}

Toute surface forestière accessible pourrait faire l'objet d'épandage, mais il faut aussi protéger l'environnement aquatique des risques de contamination par les boues. Aucune norme n'existe pour les forêts, mais celles éditées par le MENVIQ (Gouvernement du Québec, 1986b) pourraient être utilisées. Ainsi, entre $0 \%$ et $3 \%$ de pente, aucun épandage ne devrait être fait à moins de 60 mètres d'un plan ou cours d'eau. Cette distance est portée à 120 mètres et 180 mètres pour des pentes entre $3 \%$ à $6 \%$ et $6 \%$ à $9 \%$ respectivement. Finalement, aucun épandage ne devrait être effectué si les pentes sont supérieures à $9 \%$.

Afin de réduire au maximum les pertes d'éléments nutritifs par lessivage, il est recommandé d'attendre que les sols soient assez secs avant d'épandre les boues. Au printemps, cela signifie d'attendre que le sol soit ressuyé et suffisamment

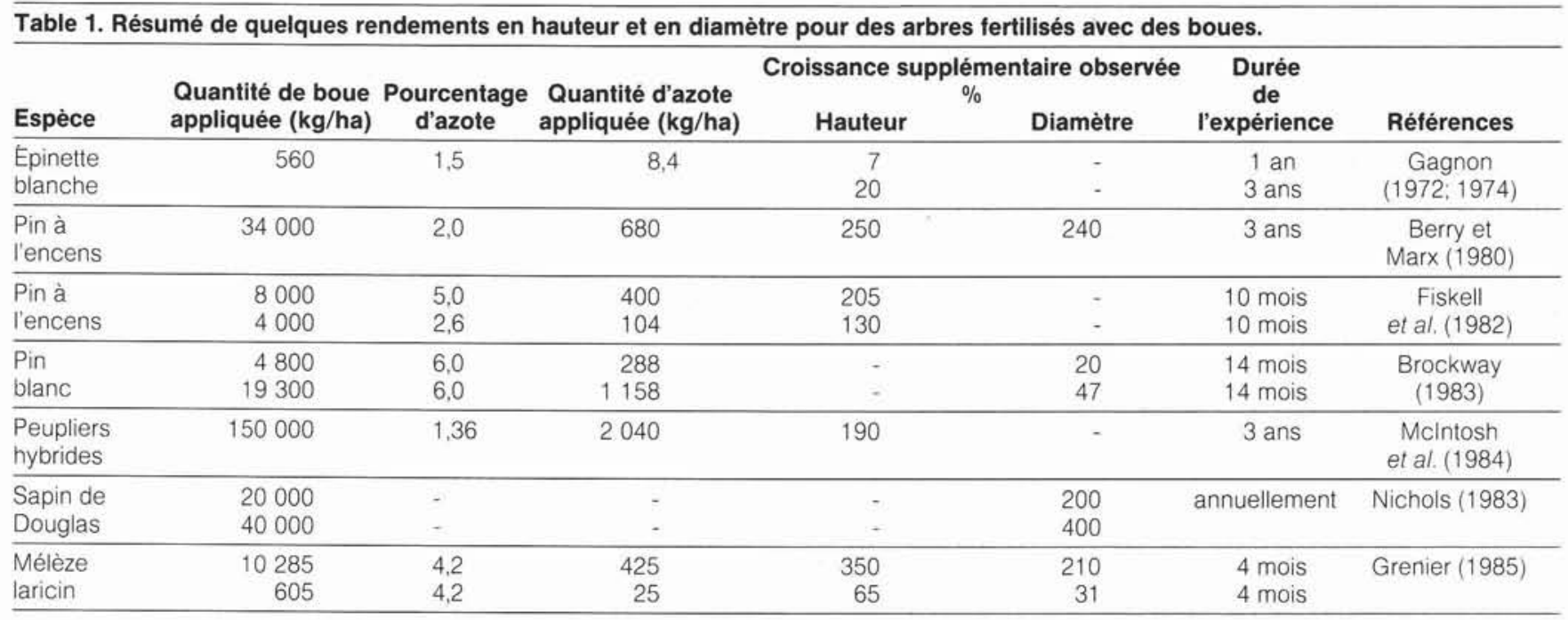



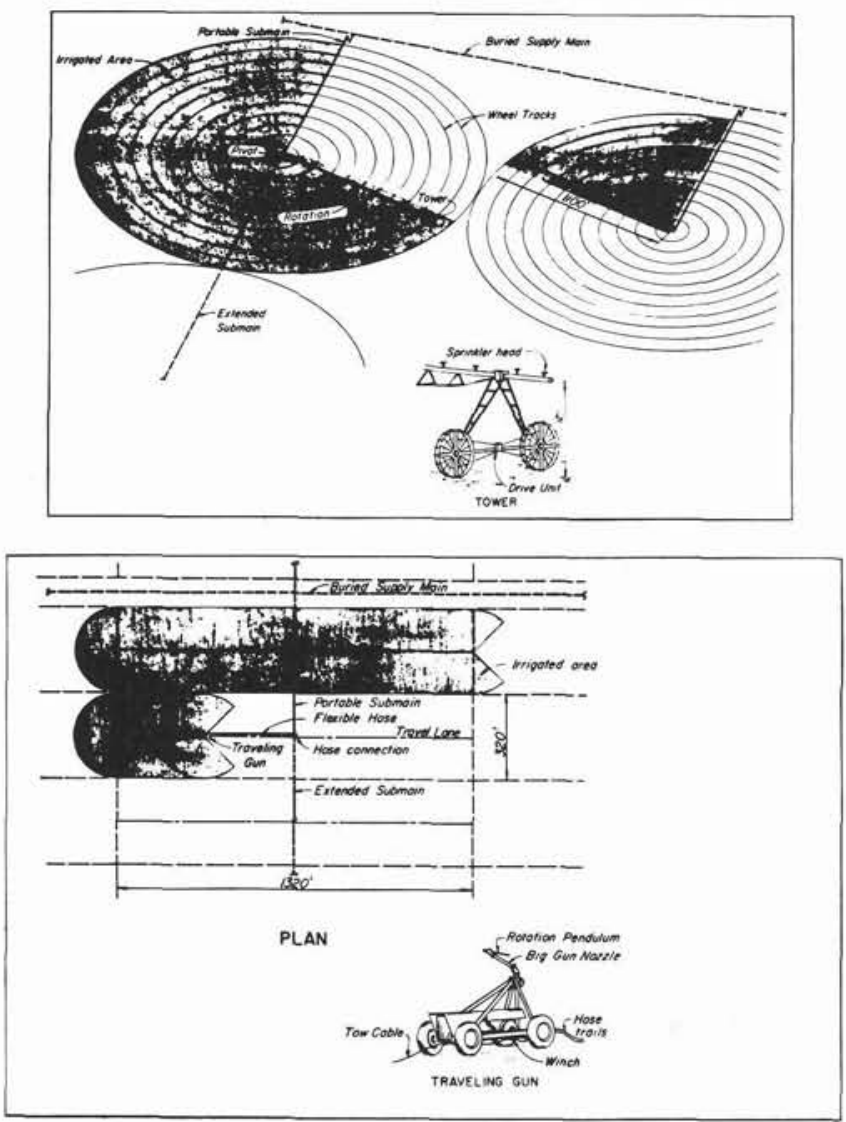

Figure 4. Gicleurs montés sur chariots mobiles.

drainé. Cela facilitera aussi le passage de la machinerie qui risque moins de s'enfoncer et de compacter le sol. Les périodes pluvieuses devraient aussi être exclues pour les mêmes raisons. Enfin, les sols gelés, enneigés ou inondés ne devraient pas recevoir de boues.

Les opérations d'épandage pourraient être couplées à d'autres travaux sylvicoles qui sont prévus dans un plan d'aménagement, tel le reboisement, l'entretien de plantations, les éclaircies, les coupes par bandes ou par damiers et les coupes totales, Cependant, ce ne sont pas tous les stades de développement (âge) de la forêt qui sont aptes à être fertilisés. Chacun est ici examiné plus en détail.

\section{Reboisement}

L'épandage avant la mise en terre des plants est l'activité la plus facile à réaliser et l'injection (ou incorporation) de la boue peut se faire sans risque d'endommager les racines (puisqu'il n'y en a pas). Cependant, si les plants étaient mis en terre immédiatement après l'épandage, deux problèmes majeurs pourraient être rencontrés. II y a d'abord des risques d'une mortalité importante, possiblement causée par un dégagement de $\mathrm{NH}_{4}^{+}$(Katayama et al., 1986; Riekerk, 1982; Le Tacon et al., 1979; Pommel, 1979; Gagnon, 1972). Le second risque est la compétition, car la fertilisation n'est pas sélective et les mauvaises herbes seraient aussi favorisées que les arbres plantés (de Vries, 1981; Berry et Marx, 1980). Cette compétition sévère exigerait des activités d'entretien (Miller, 1983): les avantages économiques retirés de la fertilisation pourraient ainsi être anéantis.

II serait possible de procéder à l'épandage des boues et de les incorporer au sol la première année, tandis que le scarifiage et la mise en terre des plants seraient faits la deuxième année. L'effet de toxicité envers les semis serait contourné et les plantes herbacées auraient emmagasiné une partie de l'azote ajouté. Le scarifiage détruirait la végétation non désirée déjà installée et l'azote capté par ces plantes serait ultérieurement restitué aux semis plantés.

\section{Entretien des plantations}

Puisque le but de cette opération est le dégagement des arbres désirés des espèces compétitives, la fertilisation par les boues résiduaires serait ici à proscrire jusqu'à ce que les arbres soient assez grands pour vaincre la compétition.

\section{Éclaircie}

L'éclaircie réduit le nombre de tiges résiduelles d'un peuplement et augmente donc par le fait même l'espacement entre elles. Cet espace, quoique n'étant pas toujours rectiligne, peut être considéré comme un chemin où une machine (type Go-Gator) pourrait circuler pour faire l'épandage. Par contre, afin de ne pas abîmer les racines des tiges résiduelles, l'épandage en surface sans injection est suggéré. Les éclaircies débutent dès que les branches des arbres se touchent, alors que les arbres encore en état juvénile exigent beaucoup de nutriments (Miller, 1981). Les éclaircies peuvent être réalisées jusqu'à la coupe totale, donc sur une grande partie de la vie du peuplement. L'épandage couplé à ces opérations sylvicoles pourrait être fait régulièrement.

\section{Coupe par bandes ou par damiers}

Cette opération, qui laisse en place de larges espaces où pourrait circuler la machinerie d'épandage, pourrait être fait de deux façons. Premièrement, dans les espaces vacants: ici, l'opération revient à celle décrite dans la section du reboisement. Deuxièmement, dans les espaces boisés résiduels: les boues pourraient être épandues en circulant près des lisières avec des véhicules de type Go-Gator

\section{Coupe totale}

La coupe totale précède une opération de reboisement. Ainsi, l'opération d'épandage à ce stade revient à celle du reboisement. Cependant, l'épandage pourrait être réalisé quelques années avant la coupe totale pour obtenir un accroissement supplémentaire en fin de révolution du peuplement forestier. Le nombre de tiges étant réduit, la circulation en forêt est facilement réalisable avec les machines spécialisées.

\section{Quantité de boues à appliquer}

La plus grande inconnue consiste à identifier les doses de boues qui devraient être appliquées à chaque type de forêt. Bien qu'il soit reconnu que l'azote est le facteur limitatif le plus important, aucune réponse précise n'est encore disponible quant aux quantités à ajouter (Mahendrappa et al., 1986). Brockway et Urie (1983) apportent une réponse indirecte à ce problème. Selon ces auteurs, il faut rechercher la dose qui minimisera les impacts négatifs liés à la fertilisation, dont le plus important est la pollution causée par le lessivage des nitrates $\left(\mathrm{NO}_{3}^{-}\right)$. Comme mesure de référence, la norme de 10 $\mathrm{mg} \mathrm{NO}_{3}^{-} / \mathrm{L}$, qui correspond au critère de qualité pour l'eau potable, a été choisie comme seuil à ne pas dépasser. Afin de la mesurer, des lysimètres sont installés en forêt dans le sol et des échantillons sont prélevés. La quantité de boue pouvant être appliquée sur un site particulier est ainsi déduite (fig. 5). 


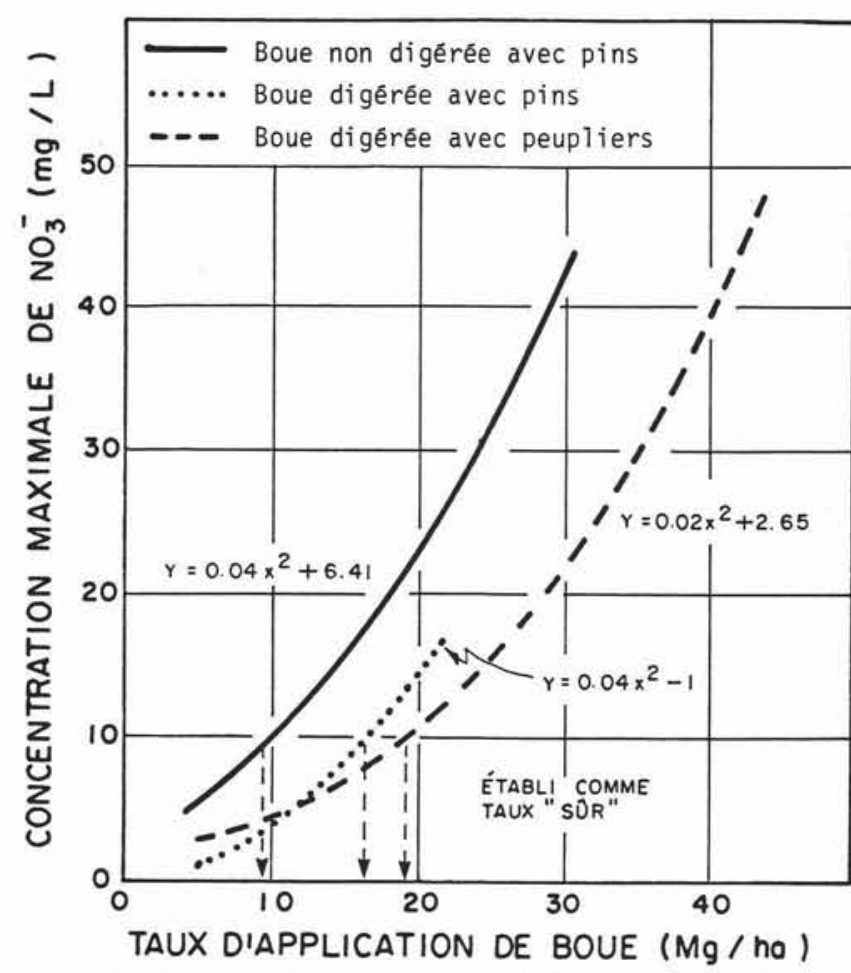

Figure 5. Courbes des concentrations résiduelles de $\mathrm{NO}_{3}$ dans les percolats selon les types de boues, les doses appliquées et la culture en place. (tiré de Brockway et Urie, 1983).

Cette méthode a l'avantage d'être simple et efficace. On maximise ainsi les chances de ne pas créer des problèmes de pollution en voulant valoriser les boues résiduaires. Concernant les périodes de récurrence, elles vont avec les travaux sylvicoles effectués. À titre indicatif, Miller (1983) rapporte qu'une intervention décennale de l'ordre de $200 \mathrm{~kg}$ $\mathrm{N} /$ ha serait réaliste, ce qui est appuyé par Mahendrappa et al. (1986) qui rapportent que les arbres à l'état juvénile captent environ $20 \mathrm{~kg} \mathrm{~N} / \mathrm{ha}$-an.

Afin de minimiser la volatilisation de $\mathrm{NH}_{3}$, le guide de valorisation agricole des boues résiduaires (Gouvernement du Québec, 1986b) rapporte que les boues devront être incorporées, soit directement lors de l'épandage, soit après l'épandage en surface, mais alors dans un délai de 24 heures. II n'est pas certain qu'une telle pratique soit souhaitable en forêt, vu que la culture est déjà en place: les risques associés à la perturbation voire la rupture des systèmes racinaires sont très importants, phénomène qui est minime pour les cultures agronomiques. Pour cette raison, en forêt l'enfouissement ne devrait pas être considéré comme une activité obligatoire. En effet, les expériences effectuées en forêt et rapportées dans la littérature se font avec des épandages en surface.

\section{Conclusion}

L'épandage en forêt est une opération réalisable: les quelques expériences rapportées précédemment démontrent le pouvoir fertilisant des boues résiduaires et l'expérience de Seattle, plus particulièrement, démontre la possibilité de faire de l'épandage à grande échelle avec de la machinerie appropriée. Le Québec dispose de vastes superficies forestières dont les rendements pourraient être augmentés en même temps que les boues produites ici seraient écoulées. Nous croyons que la valorisation des boues résiduaires en forêt est une opération qui a sa place dans la gestion intégrée du territoire et des ressources.

Par contre, il faut être prudent lors de l'utilisation de ce matériel. Idéalement, sa composition devrait être connue le plus exactement possible pour minimiser les risques environnementaux de pollution par les métaux et les composés organiques. Une boue jugée impropre à l'agriculture ne devrait pas être utilisée en foresterie. Un suivi serait aussi souhaitable pour vérifier si les eaux de surface et la nappe phréatique sont contaminées et pour évaluer si les sols et les populations fauniques et floristiques sont modifiées.

Nous nous sommes intéressés ici à la seule production de matière ligneuse, mais la porte à d'autres types de recherches reliées à l'utilisation des boues résiduaires et à ses effets sur l'environnement est toute grande ouverte.

\section{Références}

Anred. 1983. La valorisation agricole des boues de stations d'épuration. Agence nationale de récupération et d'élimination des déchets, France, 20 pages.

Baldwin, A., T.A. Brown, P.H.T. Beckett et G.E.P. Elliott. 1983. The forms of combination of $\mathrm{Cu}$ and $\mathrm{Zn}$ in digested sewage sludge. Water Res. 17: 1935-1944.

Berry, C.R. et D.H. Marx. 1980. Significance of various soil amendments to borrow pit reclamation with loblolly pine and fescue. Reclamation Review. 3: 87-94

Blake, T.J. et W.E. Raitanen. 1981. A summary of factors influencing coppicing. International Energy Agency, Program of research. development and demonstration on forestry energy. National Swedish Board for Energy Source Development, Stockholm. NE 1981: 22, $24 \mathrm{p}$

Brockway, D.G. 1983. Forest floor, soil and vegetation responses to sludge fertilization in red and white pine plantations. Soil Sci. Soc. Am. J. 47: 776-784.

Brockway, D.G. et D.H. Urie. 1983. Determining sludge fertilization rates for forests from nitrate- $\mathrm{N}$ in leachate and groundwater. J. Environ. Qual. 12: 487-492

Brockway, D.G., G. Schneider et D.P. White. 1979. Dynamics of municipal wastewater renovation in a young conifer-hardwood plantation in Michigan. In Utilization of municipal sewage effluent and sludge on forest and disturbed land. W.E. Sopper et S.N. Kerr. (Eds.) Penn. State Univ. Press, pp. 87-102.

Cole, D.W. 1981. Mineral cycling in forest ecosystems of the Pacific Northwest. In Proc. Forest fertilization conference. S.P. Gessel, R.M. Kenady et W.A. Atkinson. (Eds.) Univ. Wash., pp. 29-36.

Couillard, D. 1988. Etude de quelques indices de croissance du Larix laricina fertilisé par des boues anaérobies. Environ. Technol. Let. 9: 191-206.

Couillard, D. 1983. Quality of life: the importance of manenvironment relations and a tentative conceptual model. $\mathrm{J}$. Envir. Syst. 12: 163-185.

Couillard, D. et Y. Grenier. 1987. Alternative à la gestion des boues résiduaires municipales: recyclage en sylviculture. Sci. et Tech. de l'Eau, 20: 215-221.

Couillard, D., M. Crowley et J.L. Sasseville. 1986. Technological public choice in practice: the case of wastewater treatment facilities. J. Env. Manage. 22: 133-146.

Crites, R.W. 1984. Land use of wastewater and sludge. Environ. Sci. Technol. 18: 140A à 147A.

Crowley, M., D. Couillard et J.L. Sasseville. 1984. Le processus de choix des technologies du programme d'assainissement des eaux du Québec. Proceedings: assises annuelles de I'AQTE, pp. $173-198$.

de Vries, M.P.C. 1981. Fertilizer value of a dried sewage sludge for Pinus radiata plantations. Aust. For. 44: 190-193.

Environnement Canada. 1985. L'épandage des eaux usées traitées et des boues d'épuration d'origine urbaine. Service de la protection de l'environnement, guide SPE 6-EP-84-1, 190 pages

EPA. 1979. Sludge treatment and disposal. Process design manual. EPA-625/1-79-011, 952 pages. 
EPA. 1983. Land application of municipal sludge. Process design manual. EPA-625/1-83-016, 466 pages.

Fiskell, J.G.A., R. Rebertus, N.B. Comerford et W.L. Pritchett. 1984. Comparing soil tests for a forested acid sandy soil top dressed with sewage effluent. Soil Sci. Soc. Am. J. 48: 1170-1174.

Fiskell, J.G.A., F.G. Martin, W.L. Pritchett et M. Maftoun. 1982. Effects of cadmium levels and sludges on loblolly pine seedlings. Soils and Crop Sciences Society of Florida Proc. Vol. 41. Soil. Sci. Dept. Univ. of Florida, Gainesville, pp. 163-168.

Gagnon, J.D. 1974. Results of fertilizer experiments in Quebec. Proc. Workshop on Forest Fertilization in Canada. Sault-Ste-Marie, Ontario. Edité par Environnement Canada, Service des forêts, pp. 83-91

Gagnon, J.D. 1972. Les égouts domestiques: un engrais valable en foresterie. Édité par Environnement Canada, Centre de recherches forestières des Laurentides, Québec. Rapport QF-X-30, 13 pages.

Gagnon, P. 1985. Communication personnelle (fonctionnaire au MENVIQ)

Giroux, I. 1986. Evaluation des sites pour l'épandage de boues d'usine d'épuration. INRS-Eau, Université du Québec, mémoire de maitrise, 175 pages.

Giroux, M. 1987. Communication personnelle (fonctionnaire au MAPAQ)

Gouvernement du Québec. 1987. Communication personnelle (M.P. Gagnon, MENVIQ, Direction du contrôle budgétaire).

Gouvernement du Québec. 1986a. Rapport du groupe de travail sur le programme d'assainissement des eaux du Québec. Ministère de l'Environnement, 49 pages +8 annexes.

Gouvernement du Québec. 1986b. La valorisation agricole des boues de stations d'épuration. Ministère de l'Environnement. $47 p$

Gouvernement du Québec. 1985a. L'assainissement des eaux au Québec. Ministère de l'Environnement, 16 pages.

Gouvernement du Québec. 1985b. Allocution de M. Michel P. Lamontagne, sous-ministre adjoint, gestion et assainissement de l'eau, ministère de l'Environnement, lors de la journée perspective sur la construction, 41 e congrès annuel de L'A.C.R.G.T.Q. Le 21 février 1985, 8 pages

Gouvernement du Québec. 1984. Bilan du programme de l'assainissement des eaux. Allocution du ministre de l'Environnement, M. Adrien Ouellette lors du colloque de I'Union des municipalités du Québec sur l'assainissement des eaux. Montréal, le 7 septembre 1984, 14 pages.

Gouvernement du Québec. 1983. Document de politique sur la gestion des boues provenant de l'épuration des eaux usées. Ministère de l'Environnement, Québec, 22 pages.

Grenier, Y. 1985. La valorisation des boues d'usine d'épuration des eaux en fertilisation forestière. INRS-Eau, Université du Québec, mémoire de maîtrise, 290 pages.

Grenier, Y. et D. Couillard. 1987. Le recyclage des boues d'usine d'épuration des eaux en fertilisation forestière. J. GEOS, 16 : 22-26.

Guarino, C.F., M.D. Nelson, S.A. Townsend, T.E. Wilson et E.F. Ballotti. 1975. Land and sea solids management alternatives in Philadelphia. JWPCF. 47: 2551-2564

Hecht, N.L., D.S. Duvall et A.S. Rashidi. 1975. Characterization and utilization of municipal and utility sludges and ashes. Volume II: municipal sludges. EPA-670/2-75-033b, 231 pages.

Hornbeck, J.W. et W. Kropelin. 1982. Nutrient removal and leaching from a whole tree harvest of northern hardwoods. J. Environ. Qual. 11: 309-316

Hornbeck, J.W., M.T. Koterba et R.S. Pierce. 1979. Sludge application to a northern hardwood forest in New-Hampshire: potential for dual benefit? In Utilization of municipal sewage effluent and sludge on forest and disturbed land. W.E. Sopper et S.N. Kerr. (Eds.) Penn. State Univ. Press, pp. 137-143.

Jones, A.R.C. et J. Grant. 1983. Hybrid poplar or hardwood coppice? An agroforestry option to economically increasing wood production in Eastern Canada. For. Chron. 59: 143-145.
Katayama, A., M. Hirai, M. Shoda et H. Kubota. 1986. Factors affecting the stabilization period of sewage sludge in soil with reference to the gel chromatographic pattern. Soil Sci. Plant. Nutr. 32: 383-395.

Lester, J.N., R.M. Sterritt et P.W.W. Kirk. 1983. Significance and behaviour of heavy metals in waste water treatment processes: II. Sludge treatment and disposal. The Science of the Total Environment. 30: 45-83.

Le Tacon, F., J. Garbaye et A. Clément. 1979. Possibilité d'utilisation des boues résiduaires de stations d'épuration urbaines en sylviculture: effet sur les sols et les eaux de drainage. Proc. First European Symposium on the Treatment and Use of Sewage Sludge, Cadarache 13-15 February 1979. Édité par D. Alexandre et H. Ott, pp. 291-308.

Mahendrappa, M.K., N.W. Foster, G.F. Weetman et H.H. Krause. 1986. Nutrient cycling and availability in forest soils. Can. J. Soil. Sci. 66: 547-572.

McIntosh, M.S., J.E. Foss, D.C. Wolf, K.R. Brandt et R. Darmody. 1984. Effect of composted municipal sewage sludge on growth and elemental composition of white pine and hybrid poplar. J. Environ. Qual. 13: 60-62.

Metro. 1983a. Sludge management plan. Municipality of Metropolitan Seattle.

Metro. 1983b. Draft plan: Pilchuck tree farm; demonstration sludge application project. Municipality of Metropolitan Seattle. 140 p.

Miller, H.G. 1983. Wood energy plantations: diagnosis of nutrient deficiencies and the prescription of fertilizer applications in biomass production. Édité par International Energy Agreement - Forestry Energy Agency. Rapport no 3, Program Group "B", $20 \mathrm{p}$

Miller, H.G. 1981. Forest fertilization: some guiding concepts. For. 54 : 157-167.

Möller, G. 1974. Aspects pratiques et économiques de la fertilisation des forêts. Phosphore et Agriculture. 62: 35-51.

Morrison, I.K. 1974. Mineral nutrition of conifers with special reference to nutriment status interpretation: a review. Service canadien des forêts, Centre de recherches forestières des Grands-Lacs. Publication no 1344.74 p.

Nichols, C. 1980. Engineering aspects of dewatered sludge land application to forest soils. Thèse de maîtrise, Université de Washington, 84 pages.

Nichols, C. 1983. Seattle sludge and silviculture. Water Engineering and Management. 130: 36-37.

Patrick, J.H. et D.W. Smith. 1975. Forest management and nutrient cycling in eastern hardwoods. USDA For. Serv. N.E. Forest Exp. Stn. Upper Darby, PA - Paper N.E.-326. 12 p.

Pommel, B. (1979). La valorisation agricole des déchets: 2) Les boues résiduaires urbaines Institut national de la recherche agronomique (INRA). Station d'agronomie de Bordeaux, 70 pages

Riekerk, H. 1982. How much sewage nitrogen on forest soils? A case history. BioCycle. 23: 53-56.

Roberge, M.R., J.D. Gagnon et C.H. Ung. 1980. Essai d'un dosage d'azote dans trois sapinières de 60 ans au Québec. Résultats de 10 ans. Can. J. For. Res. 10: 470-475

Sabey, B.R. et W.E. Hart. 1975. Land application of sewage sludge: 1. Effect on growth and chemical composition of plants. J. Environ. Qual. 4:252-256.

Sidle, R.C. et L.T. Kardos. 1977. Transport of heavy metals in a sludge-treated forested area. J. Environ. Qual. 6: 431-437.

Vergès, G. 1984. La disposition des boues des stations d'épuration. Colloque sur l'assainissement des eaux. U.M.Q., septembre 1984, 11 pages

Vézina, P.E. et M.R. Roberge. 1981. Comment aménager nos forêts. Les Presses de l'Université Laval, 273 pages.

Webber, M.D. 1984. Épandage des boues résiduaires sur les sols: une évaluation. Agriculture Canada, direction générale de la recherche, 45 pages. 